\title{
Unigrid for Multigrid Simulation
}

\author{
By S. F. McCormick and J. W. Ruge
}

\begin{abstract}
This paper develops unigrid which is an algorithm that results from interpreting multigrid as it directly affects the fine grid approximation. Unigrid is theoretically equivalent to multigrid under certain assumptions, yet has different computational characteristics. Though it is generally less efficient, unigrid is very useful as a software tool for testing the feasibility of applying multigrid to a given problem. This is illustrated with several numerical examples.
\end{abstract}

1. Introduction. The subject of this paper is unigrid, a method designed primarily for solving the equations arising from discretization of partial differential boundary value problems. The method is closely related and, under certain conditions, theoretically equivalent to multigrid, although all of the unigrid computations are performed on a single grid, as the name implies. Unigrid is generally more computationally costly than multigrid, yet its simplicity is reflected in its relative ease of code implementation and use with existing applications software. This enables unigrid to act effectively as a software tool to simulate the multigrid approach that requires a more intensive design effort.

Because of its conceptual simplicity, in a limited way the ideas basic to unigrid can be understood independently from those of multigrid. In fact, unigrid is described below as a directional iteration method and can therefore be considered from a purely algebraic point of view. However, the ability to use unigrid effectively depends on an understanding of the discretization and relaxation principles that are the foundation for multigrid. Simplicity of description should not compromise these principles, so it is advised that the reader have a basic understanding of multigrid (cf. [1]).

On the other hand, for the reader that does not have this understanding, and for general concreteness, the next section contains a simplified description of unigrid applied to a one-dimensional model problem, However, the remaining sections assume familiarity with the concepts and notation of multigrid and include the general development of unigrid (Section 3); its relationship to multigrid (Section 4); some theory (Section 5); descriptions of several versions of unigrid together with code and output listings for a few that were implemented in BASIC on a HewlettPackard 9845B (Section 6); and the use of unigrid illustrated with some experiments on irregular boundaries and nonlinearities (Section 7).

Received July 10, 1981; revised January 8, 1982.

1980 Mathematics Subject Classification. Primary 65N99; Secondary 65N20, 65F10.

(C) 1983 American Mathematical Society $0025-5718 / 83 / 0000-1239 / \$ 05.50$ 
2. A Simple Example. To describe unigrid in its simplest form, consider the two-point boundary value problem

$$
\begin{aligned}
-y^{\prime \prime}(x) & =f(x), \quad x \in[0,1], \\
y(0) & =y(1)=0 .
\end{aligned}
$$

Assuming that $h=2^{-m}$ for some positive integer $m$, let $n=2^{m}-1$, define the grid points by $x_{k}=k h, 1 \leqslant k \leqslant n$, and suppose that

$$
A^{h} U^{h}=f^{h}, \quad U^{h} \in R^{n},
$$

denotes a suitable discrete approximation to (2.1). For example, the components of $f^{h}$ might correspond to $f$ evaluated at the grid points, and $A^{h}$ might denote the usual $n$ by $n$ tridiagonal central difference matrix with diagonal entries $2 h^{-2}$ and off-diagonal entries $-h^{-2}$.

In what follows, upper and lower case letters will denote, respectively, exact and approximate solutions of (2.2).

The $k$ th step of one sweep of Gauss-Seidel relaxation can now be written as

$$
u^{h} \leftarrow u^{h}-\frac{\left\langle r^{h}, e_{k}^{h}\right\rangle}{\left\langle A^{h} e_{k}^{h}, e_{k}^{h}\right\rangle} e_{k}^{h} .
$$

Here, $e_{k}^{h}$ is the $k$ th column of the $n$ by $n$ identity matrix, the residual is given by $r^{h}=A^{h} u^{h}-f^{h}$, and the left arrow denotes replacement, thereby avoiding iteration subscripts.

Heuristically speaking, the main difficulty with Gauss-Seidel applied to (2.2) stems from its restriction to the use of local data. More specifically, information (i.e., residual error) at grid points distant from $x_{k}$ takes many sweeps to properly affect $u_{k}^{h}$. Thus, the "spiked" grid functions $e_{k}^{h}$ depicted in Figure 1 do little to provide communication between widely separated grid points.

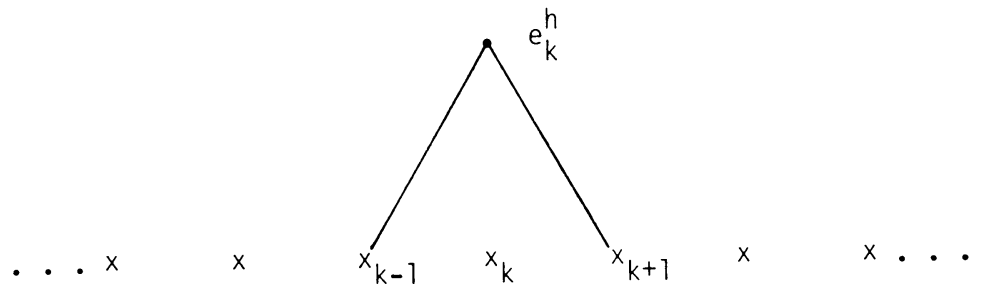

FIGURE 1

This interpretation suggests the use of "broader-based" directions such as those defined recursively by

$$
d_{k}^{H}=\left\{\begin{array}{lc}
.5 d_{2 k-1}^{H / 2}+d_{2 k}^{H / 2}+.5 d_{2 k+1}^{H / 2}, & H=2^{i}, 1 \leqslant i \leqslant m-1, \\
e_{k}^{h}, \quad H=h, 1 \leqslant k \leqslant n . & 1 \leqslant k \leqslant 2^{i}-1,
\end{array}\right.
$$

These directions are illustrated in Figure 2 for the case $m=3$. Note, in general, that these directions for level $H$ are simply the grid functions $e_{k}^{H}$ (defined on grid points $x_{2^{\prime} k}^{h}$ ) linearly interpolated to grid $h$. 

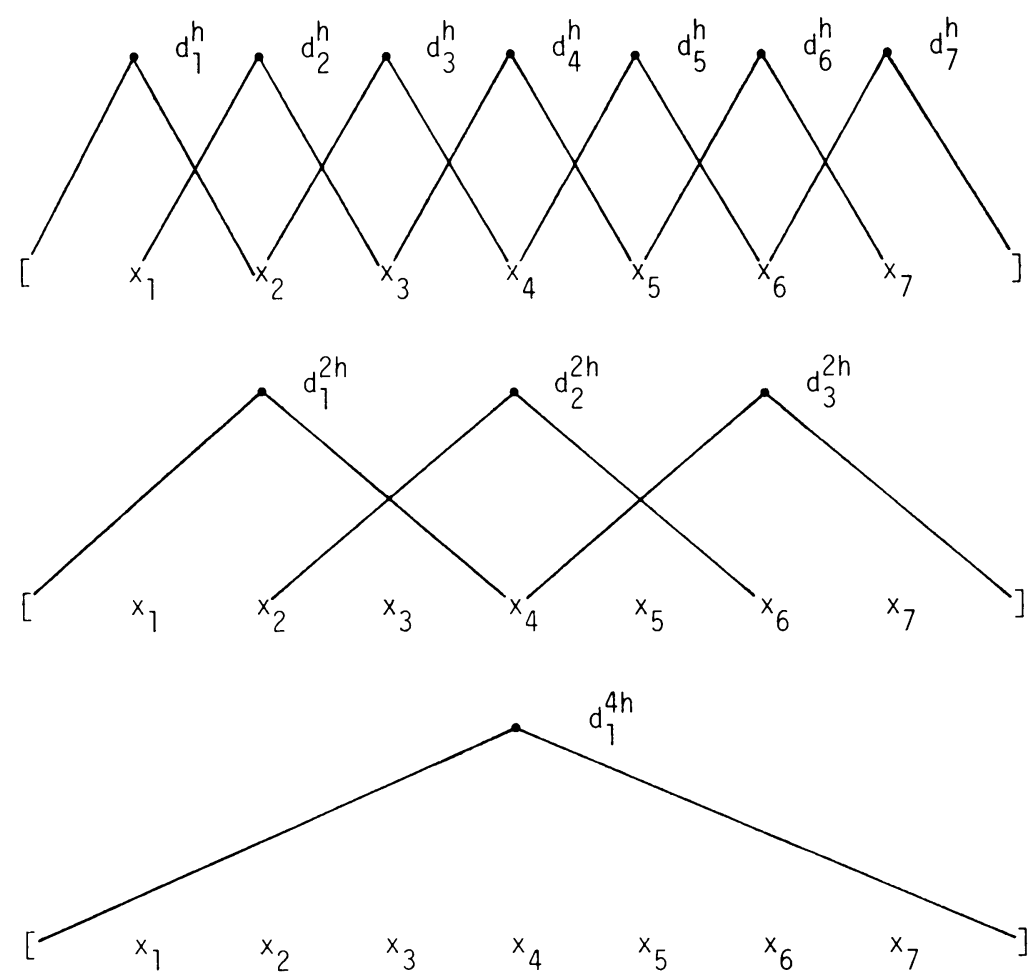

FIGURE 2

To use these directions, as with Gauss-Seidel, the objective is to subtract from $u^{h}$ a proper multiple of $d_{k}^{H}$ so that the new residual is orthogonal to $d_{k}^{H}$. This is given algebraically by the directional iteration

$$
u^{h} \leftarrow u^{h}-\frac{\left\langle r^{H}, d_{k}^{H}\right\rangle}{\left\langle A^{h} d_{k}^{H}, d_{k}^{H}\right\rangle} d_{k}^{H} .
$$

A simple version of unigrid can then be described in terms of one full sweep over $k$ and $H$ as follows:

For each $i=n, n-1, \ldots, 1$, let $H=2^{-i}$ and $n^{H}=2^{i}-1$ and iterate via (2.5) for each $k=1,2, \ldots, n^{H}$.

Note that unigrid in this form consists of the directional iteration (2.5) with the $d_{k}^{H}$ cycling in order through the sequence

$$
\left(d_{1}^{h}, d_{2}^{h}, \ldots, d_{n^{h}}^{h} ; d_{1}^{2 h}, d_{2}^{2 h}, \ldots, d_{n^{2 h}}^{2 h} ; \ldots ; d_{1}^{1 / 4}, d_{2}^{1 / 4}, d_{3}^{1 / 4} ; d_{1}^{1 / 2}\right) .
$$

For a fixed "level" of these directions specified by the superscript $H$, this process attempts to reduce the residual error in $S_{H}=\operatorname{span}\left\{d_{k}^{H}: 1 \leqslant k \leqslant n^{H}\right\}$. Analogous to the discussion above for $H=h$, it is effective only in terms of the transmission of information that is local relative to $S_{H}$. Specifically, data from a point that is further away than a few directions is slow to propagate via (2.5). (Note in Figure 2 that $x_{1}$ and $x_{7}$ are distant relative to level $h$ but near relative to level $4 h$.) Errors due to relatively long distance data connections can be quickly reduced only on the broader 
levels $H^{\prime}=2 H, 4 H, \ldots$ (Note that $S_{H}$ is a proper subset of $S_{H / 2}$ so that unigrid converges here only because error components in the complement of $S_{H}$ are reduced on the "narrower" levels $H^{\prime}=H / 2, H / 4, \ldots$ )

The next section generalizes these concepts in the framework of conventional multigrid notation.

3. The General Case. As in [2], assume for focus that the problem to be solved is the $d$-dimensional operator equation

$$
A U=f, \quad U \in \mathcal{H}_{1},
$$

where $A: \mathscr{H}_{1} \rightarrow \mathscr{H}_{2}$ is a linear operator and $\mathscr{H}_{1}$ and $\mathscr{H}_{2}$ are appropriate Hilbert spaces of functions defined on a region $\Omega$ in $R^{d}, d \geqslant 2$. Assume that (3.1) admits discretizations by a family of matrix equations, parameterized by admissible grid sizes $h>0$ and given by

$$
A^{h} U^{h}=f^{h}, \quad U^{h} \in \mathcal{H}^{h},
$$

where $\mathcal{H}^{h}=R^{n^{h}}$ and $n^{h}$ is an integer approximately proportional to $h^{-d}$. (Note that $n^{h}=h^{-1}-1$ for the example in the previous section.) The grid transfers are full rank linear operators, represented by $I_{h}^{h}: \mathscr{H}^{\bar{h}} \rightarrow \mathscr{H}^{h}$, that satisfy the consistency condition $I_{h}^{h}=I_{h^{\prime}}^{h} I_{\bar{h}}^{h^{\prime}}$ for admissible $\bar{h}, h^{\prime}, h$ when $\bar{h}<h^{\prime}<h$ or $\bar{h}>h^{\prime}>h$.

The class of relaxation methods considered here can be written in terms of the directional iteration operator $G^{h}: \mathcal{H}^{h} \times \mathcal{H}^{h} \rightarrow \mathscr{H}^{h}$ which, with relaxation parameter $\omega$ and residual $r^{h}=A^{h} u^{h}-f^{h}$, is given by

$$
G^{h}\left(u^{h}, d^{h}\right)=u^{h}-\omega \frac{\left\langle r^{h}, d^{h}\right\rangle}{\left\langle A^{h} d^{h}, d^{h}\right\rangle} d^{h} .
$$

The relaxation process is then specified by an ordered set of directions $\left.{ }^{1}\right)^{h}=$ $\left(\delta_{1}^{h}, \delta_{2}^{h}, \ldots, \delta_{n^{h}}^{h}\right) \subset \mathcal{H}^{h}$, so that one sweep with the initial guess $u^{h}$ consists of iterating via

$$
u^{h} \leftarrow G^{h}\left(u^{h}, d^{h}\right)
$$

as $d^{h}$ varies over $\mathscr{Q}^{h}$. (Note that Gauss-Seidel is specified by the choice $\delta_{k}^{h}=e_{k}^{h}$, and with some modification this development extends to more general relaxation schemes including Jacobi.)

To define unigrid for a given admissible grid size $h=H_{0}$, suppose $m \geqslant 1$ is an integer so that $H_{q}=2^{q} H_{0}$ are admissible, $q \leqslant m$, and define the direction sets according to

$$
\mathcal{Q}_{q}^{h}=\left(d_{1}^{H_{q}}, d_{2}^{H_{q}}, \ldots, d_{n^{H_{q}}}^{H_{q}}\right), \quad 0 \leqslant q \leqslant m,
$$

where $d_{k}^{H_{q}}=I_{H_{q}}^{h} d_{k}^{H_{q}}$. Thus, the directions on level $q$ are just the relaxation directions on grid $H_{q}$ transferred to grid $h=H_{0}$.

With relaxation and cycling parameters $\mu, \nu$ and $\nu_{c}$ and present approximation $u^{h}$, then one of the many possible unigrid schemes is defined recursively by:

One unigrid cycle on level $q \geqslant 0$ consists first of $\nu$ unigrid relaxation sweeps via (3.4) using the ordered directions $d_{k}^{H_{q}}, k=1,2, \ldots, n^{H_{q}}$, followed for $q<m$ by $\mu$ cycles on level $q+1$ and for $q=m$ by $\nu_{c}$ additional sweeps via (3.4).

One complete unigrid cycle applied to (3.2) on the finest level $h=H_{0}$ with right-hand side $f^{h}$ and present approximation $u^{h}$ will be denoted by $\mathrm{UG}^{h}\left(u^{h}, f^{h}\right)$. 
Remark 1. Except possibly for determining the direction, as the name implies, unigrid operations are performed on grid $h=H_{0}$ only. Since the directions depend not directly on the operator $A$, but rather on the domain $\Omega$, then there are usually several ways for determining them without explicit appeal to the coarser grids $H_{q}$, $q>0$. The simplest situation in two dimensions is for a rectangular domain $\Omega$ where the discretization allows us to define $I_{2 h}^{h}$ as linear interpolation. With Gauss-Seidel as the fine grid relaxation, for example, and with the usual double subscript notation and $n^{h}=N^{h} \times N^{h}$, then $d_{k, l}^{h}=e_{k, l}^{h}$ for $h=H_{0}, 1 \leqslant k, l \leqslant N^{h}$, and the coarse grid directions are defined recursively for $h=H_{q}$ by

$$
d_{k, l}^{2 h}=\sum_{i, j=-1}^{1} 2^{2-(|i|+|j|)} d_{2 k+i, 2 l+j}^{h}
$$

Note that unigrid is invariant under a change of scale for these directions, allowing for the coefficients in (3.6) to be integers. Another of the many ways to define these directions is algebraically in terms of the $i, j$ component of $d_{k . l}^{H_{q}}$ as in

$$
d_{k, l}^{H_{u}}(i, j)=\left\{\begin{array}{l}
\left(2^{q}-|k-i|\right)\left(2^{q}-|l-j|\right), \quad|k-i|,|l-j| \leqslant 2^{q} \\
0, \quad \text { otherwise. }
\end{array}\right.
$$

This is the approach taken in the experiments reported in Section 6.

4. Relationship to Multigrid. The unigrid cycle defined in Section 3 corresponds to the multigrid correction scheme (cf. [1]). One such multigrid cycle on problem (3.2) with present approximation $u^{h}$, right-hand side $\bar{f}^{h}$, and $h=H_{q}$ is denoted by $\mathrm{MG}_{h}\left(u^{h}, \bar{f}^{h}\right)$ and defined recursively by:

For $q=m, \mathrm{MG}_{h}\left(u^{h}, \bar{f}^{h}\right)$ consists of $\nu+\nu_{c}$. relaxation sweeps via (3.4) with directions $\delta_{k}^{h}, k=1,2, \ldots, n^{n}$.

For $q<m, \mathrm{MG}_{h}\left(u^{h}, \bar{f}^{h}\right)$ consists of:

(1) Perform $\nu$ relaxation sweeps via (3.4) with directions $\delta_{k}^{h}, k=1,2, \ldots, n^{h}$.

(2) Let $r^{h}=\bar{f}^{h}-A^{h} u^{h}, r^{2 h}=I_{h}^{2 h} r^{h}, u^{2 h} \leftarrow 0$, and perform $\mu$ grid $2 h$ cycles via $\mathrm{MG}_{2 h}\left(u^{2 h}, \bar{f}^{2 h}\right)$ with $\bar{f}^{2 h}=r^{2 h}$.

(3) Set $u^{h} \leftarrow u^{h}+I_{2 h}^{h} u^{2 h}$.

Note that $\bar{f}^{h}$ for $q>1$ is the residual transferred from a finer grid approximation and $u^{h}$ is an approximation to the actual error in that fine grid solution. (For $q>1$, $u^{h}$ is not an approximation to $U^{h}$, the solution of (3.2), since the right-hand side is $f^{h}$ only when $h=H_{0}$.)

There are two main differences between the unigrid and multigrid techniques described. First, because only one grid is used in unigrid, its approximate solution reflects coarse level changes immediately. Thus, the corrected solution is automatically used in later computations. In the multigrid method, however, the fine grid residual is fixed at the time that the cycle progresses to coarser grids. Thus, the computations made for the coarse grid problem are not immediately used to improve the fine grid solution and update the residual. Second, to compute the low frequency corrections, unigrid uses the full solution rather than the coarse grid representation of fine grid error that MG uses.

Ignoring computational work per step, then unigrid seems preferable to multigrid since all available information is used at every stage of the method. However, in this 
section it will be shown that these two approaches are in fact theoretically equivalent provided the MG formulation of the grid operators and transfers satisfies the variational conditions

$$
\begin{gathered}
A^{2 h}=I_{h}^{2 h} A^{h} I_{2 h}^{h}, \\
I_{h}^{2 h}=\left(I_{2 h}^{h}\right)^{T} .
\end{gathered}
$$

(Actually, (4.2) need only hold up to some scale factor which we omit for simplicity.)

THEOREM 1. Under these conditions and for the same initial approximation, $u^{h}$, right-hand side, $f^{h}$, and cycling parameters, $\nu, \nu_{c}$, and $\mu$, then $\mathrm{UG}^{h}\left(u^{h}, f^{h}\right)$ and $\mathrm{MG}^{h}\left(u^{h}, f^{h}\right)$ produce the same new approximation, that is, they are theoretically equivalent.

Proof. First consider the following algorithm, intermediate to UG and MG, which for this proof is called immediate replacement multigrid. Using $q$ here in place of $H_{q}$ for superscripts and subscripts, then one cycle on grid $H_{q}$ is denoted by $\operatorname{MGIR}_{q}\left(u^{0}, f^{0}\right)$ and is defined recursively by the following. (Note that MGIR is the same as MG except that each change in the coarse grid correction is immediately reflected in the fine grid approximation which is then used to update the fine grid residual and coarse grid equation.)

For $q=m, \operatorname{MGIR}_{q}\left(u^{0}, f^{0}\right)$ consists of $\nu+\nu_{0}$ relaxation sweeps via

$$
u^{0} \leftarrow u^{0}+\omega \frac{\left\langle I_{0}^{q}\left(f^{0}-A^{0} u^{0}\right), \delta_{k}^{q}\right\rangle}{\left\langle A^{q} \delta_{k}^{q}, \delta_{k}^{q}\right\rangle} I_{q}^{0} \delta_{k}^{q}, \quad k=1,2, \ldots, n^{q} .
$$

For $q<m$, $\operatorname{MGIR}_{q}\left(u^{0}, f^{0}\right)$ consists of $\nu$ relaxation sweeps via (4.3) followed by $\mu$ level $q-1$ cycles via $\operatorname{MGIR}_{q-1}\left(u^{0}, f^{0}\right)$.

Note that the immediate fine grid correction is incorporated in the relaxation scheme. This scheme on a level $q>0$ is just (3.3) with $u^{p}=0,0<p \leqslant q$, and $r^{q}=I_{0}^{q}\left(f^{0}-A^{0} u^{0}\right)$ followed by interpolation of the correction directly to the finest grid.

To compare MG and MGIR, consider a fine grid function, $\bar{u}^{0}$, that monitors and incorporates each MG coarse grid computation, that is, $\bar{u}^{0}$ is the function that would result if $\mathrm{MG}$ were terminated anytime in the cycle and all coarse grid corrections were interpolated through each finer grid to properly correct the finest grid approximation $u^{0}$. (The corresponding function for MGIR is simply $u^{0}$.) In MG, assume relaxation is being performed on level $k>0$. Let $\tilde{u}^{q}$ denote the current level $q$ solution approximation for $0 \leqslant q \leqslant k$, and let $\bar{u}^{q}$ be the level $q$ monitoring approximation that incorporates the corrections from coarser grids. Thus, $\bar{u}^{k}=\tilde{u}^{k}$ and $\bar{u}^{q}=\tilde{u}^{q}+I_{q+1}^{q} \bar{u}^{q+1}$ for $q<k$. Now suppose MG and MGIR are started with the same fine grid approximation, and consider the effect of one directional iteration during a relaxation sweep on level $k>0$. In MGIR, the result of that iteration is

$$
u_{\text {new }}^{0}=u_{\text {old }}^{0}+\omega \frac{\left\langle I_{0}^{k}\left(f^{0}-A^{0} u_{\text {old }}^{0}\right), d^{k}\right\rangle}{\left\langle A^{k} d^{k}, d^{k}\right\rangle} I_{k}^{0} d^{k}
$$


In MG, a change of $\alpha d^{k}$ in $\tilde{u}^{k}$ results in a change of $\alpha I_{k}^{0} d^{k}$ in $\bar{u}^{0}$, so the corresponding change for $\mathrm{MG}$ is

$$
\bar{u}_{\text {new }}^{0}=\bar{u}_{\text {old }}^{0}+\omega \frac{\left\langle r^{k}, d^{k}\right\rangle}{\left\langle A^{k} d^{k}, d^{k}\right\rangle} I_{k}^{0} d^{k} .
$$

Using the recursive definitions $r^{q}=f^{q}-A^{q} \tilde{u}^{q}$ and $\bar{u}^{q}=\tilde{u}^{q}+I_{q+1}^{q} \bar{u}^{q+1}$, then the MG monitoring residual satisfies

$$
r^{k}=f^{k}-A^{k} \bar{u}^{k}=I_{0}^{k}\left(f^{0}-A^{0} \bar{u}_{\text {old }}^{0}\right)+\sum_{q=1}^{k} I_{q}^{k}\left(I_{q-1}^{q} A^{q-1} I_{q}^{q-1}-A^{q}\right) \bar{u}^{q} .
$$

By assumption (4.1), the second term in the above vanishes so that substitution into (4.5) yields

$$
\bar{u}_{\text {new }}^{0}=\bar{u}_{\text {old }}^{0}+\omega \frac{\left\langle I_{0}^{k}\left(f^{0}-A^{0} \bar{u}_{\text {old }}^{0}\right), d^{k}\right\rangle}{\left\langle A^{k} d^{k}, d^{k}\right\rangle} I_{k}^{0} d^{k} .
$$

If both algorithms begin with the same approximation, then, by induction, (4.4) and (4.6) imply that $u^{0}=\bar{u}^{0}$ at termination. Thus, assumption (4.1) ensures that MG and MGIR are equivalent.

To compare MGIR and unigrid, note that the unigrid directional iteration corresponding to (4.4) is given by

$$
u_{\text {new }}^{0}=u_{\text {old }}^{0}+\omega \frac{\left\langle f^{0}-A^{0} u_{\text {old }}^{0}, I_{k}^{0} d^{k}\right\rangle}{\left\langle A^{0} I_{k}^{0} d^{k}, I_{k}^{0} d^{k}\right\rangle} I_{k}^{0} d^{k} .
$$

Assumption (4.2) then implies that the unigrid iteration is just

$$
u_{\text {new }}^{0}=u_{\text {old }}^{0}+\omega \frac{\left\langle I_{0}^{k}\left(f^{0}-A^{0} u_{\text {old }}^{0}\right), d^{k}\right\rangle}{\left\langle I_{0}^{k} A^{0} I_{k}^{0} d^{k}, d^{k}\right\rangle} I_{k}^{0} d^{k},
$$

which, with (4.1), becomes

$$
u_{\text {new }}^{0}=u_{\text {old }}^{0}+\omega \frac{\left\langle I_{0}^{k}\left(f^{0}-A^{0} u_{\text {old }}^{0}\right), d^{k}\right\rangle}{\left\langle A^{k} d^{k}, d^{k}\right\rangle} I_{k}^{0} d^{k} .
$$

This is identical to (4.4), implying that unigrid and MGIR are equivalent methods. It follows that unigrid and multigrid are theoretically equivalent, so the proof of Theorem 1 is complete.

Although (variationally formulated) multigrid is equivalent to unigrid in that it produces the same results, there are great differences in implementation and efficiency. There are many possible implementations of unigrid with widely ranging computational characteristics, so it is difficult to make explicit comparisons, but some general differences are evident:

(1) The computational complexity of one multigrid cycle with $\mu \leqslant 3$ is $O(N)$, where $N$ is the number of fine grid points. Unigrid cycles are significantly more expensive with complexity $O(N \log N)$ for $\mu=1$ and $O\left(N^{2}\right)$ for $\mu=2$, provided some algorithmic modifications are made.

(2) In terms of storage, unigrid essentially requires only a vector of length $N$, although a trade-off with efficiency can be made by providing more storage for the function $f$ or for the directions. Usual multigrid algorithms require somewhat more 
storage than this; however, the difference between unigrid and multigrid storage requirements is not generally significant.

(3) Unigrid code is typically very compact, partly because it lacks the modular structure of multigrid software. This is one reason that unigrid code can be developed very quickly. Also, there are fewer design choices with unigrid, since the coarse grid and grid transfer operators are automatically determined. This also adds to ease of programming, but restricts flexibility of the method. The design of unigrid also guarantees convergence independently of the choice of the coarse level iteration directions and cycling scheme, so mistakes may slow convergence but do not result in divergence as often as for multigrid. This may not be an asset, however.

(4) This ease of programming and small program size make unigrid an effective method to test the convergence behavior of multigrid for many application problems. It can easily be used to replace the usual relaxation or direct solvers in existing programs in order to perform such a feasibility test. Of course, the amount of work involved makes any comparison of solution time meaningless, but actual multigrid efficiency can be determined by applying the usual multigrid operation counts to the unigrid cycling scheme. Since the methods are equivalent in terms of results when multigrid is implemented according to (4.1)-(4.2), then unigrid will accurately represent the numerical performance of such a variationally formulated multigrid scheme.

(5) As with multigrid, unigrid can be extended to apply to a broader class of problems. For example, for nonlinear problems the linear relaxation in (3.4) can be replaced by the more general procedure that changes $u^{h}$ by subtracting from it a proper multiple of $d^{h}$ so that the new residual is orthogonal to $d^{h}$. (This will usually, but not always, require the use of an inner-loop scalar nonlinear solver such as Newton's method.) This extension is simpler than the nonlinear extensions of multigrid (e.g., Newton-MG and FAS) and generally more effective in accounting for the nonlinearity since all multigrid versions freeze at least some aspect of the nonlinearity during coarse grid correction.

5. Theory. For the moment, assume that $A^{h}$ is symmetric and positive definite for all admissible $h$. Define the energy inner-product and norm on $\mathcal{H}^{h}$ by

$$
\left\langle x^{h}, y^{h}\right\rangle_{A^{h}}=\left\langle A^{h} x^{h}, y^{h}\right\rangle
$$

and

$$
\left\|x^{h}\right\|_{A^{h}}=\left\langle A^{h} x^{h}, x^{h}\right\rangle^{1 / 2},
$$

respectively. Let $\mathscr{W} \int_{\lambda}^{h}$ denote the set of all $A^{h}$-unit vectors in $\operatorname{span}\left\{w^{h}: A^{h} w^{h}=\mu w^{h}, \mu\right.$ $\leqslant \lambda\}$, and let $\mathcal{T}_{\lambda}^{h}$ denote its $A^{h}$-orthogonal complement. Let $\mathcal{G}^{h}$ signify one pass of (3.4) over the fine grid directions $\mathscr{D}_{0}^{h}$, and assume $\mathscr{D}_{0}^{h}$ spans $\mathcal{H}^{h}$. For each integer $\nu \geqslant 1$, define $\mathcal{G}_{\lambda, \nu}^{h}$ as the restriction of

$$
\left(A^{h}\right)^{-1 / 2}\left(\left(\mathcal{G}^{h}\right)^{\nu}\right)^{T} A^{h}\left(\mathcal{G}^{h}\right)^{\nu}\left(A^{h}\right)^{-1 / 2}
$$

to $\mathcal{T}_{\lambda}^{h}$. (For Jacobi-type versions of (3.4), this latter operator simplifies to $\left(\mathcal{G}^{h}\right)^{2 \nu}$.) Then, with $2 m$ the degree of the differential operator in (3.1), assume (cf. [2]):

Al. There exist constants $a>0$ and $c_{0}<\infty$ independent of $h$ such that

$$
\left\|w^{h}-R\left(I_{2 h}^{h}\right)\right\|_{A^{h}}^{2} \leqslant c_{0}\left(\lambda h^{2 m}\right)^{a}
$$


for all admissible $h$ and all $w^{h} \in \mathcal{Q} \int_{\lambda}^{h}$, where $R\left(I_{2 h}^{h}\right)$ is the range of $I_{2 h}^{h}$. (Note that $R\left(I_{2 h}^{h}\right)$ is the span of the coarse grid directions, $\mathscr{D}_{1}^{h}$.)

A2. There exist constants $c_{1}, c_{2}>0$ with $c_{1}<1$ and $c_{2} \leqslant\left(c_{1}+1\right) h^{-2 m} / \rho\left(A^{h}\right)$, where $\rho\left(A^{h}\right)$ is the spectral radius of $A^{h}$, such that

$$
\rho\left(\mathcal{G}_{\lambda, \nu}^{h}\right) \leqslant \max \left\{c_{1}^{\nu},\left|1-c_{2} \lambda h^{2 m}\right|^{\nu}\right\} .
$$

THEOREM 2. Suppose $\mu>1$ and $m$ and $\nu_{c}$ are such that the error from the coarsest level does not significantly contribute to the finest level error. Then there exists a $\nu$ independent of $h$ such that unigrid converges to the solution of (3.2) by a fixed linear rate independent of $h$.

Proof. This theorem follows from the results of Section 4 that relate unigrid to multigrid and from the theory of [2] slightly modified to account for the class of relaxation methods depicted in (3.4). This is a fairly straightforward proof so it is omitted.

Remark 2. A general nonsingular matrix problem can be transformed to a symmetric one via the normal equations

$$
\left(A^{h}\right)^{T} A^{h} U^{h}=\left(A^{h}\right)^{T} f^{h} .
$$

This is ill-advised for most methods of solution since it worsens the condition number of the problem in (3.2), but for unigrid (and multigrid) the disadvantages are usually not very great. Suppose, for example, that $A^{h}$ is symmetric to begin with and that Jacobi's method with optimal relaxation parameter $\omega$ yields a smoothing rate of $\varepsilon \leqslant 0.6$, say. Then it is relatively easy to show that the smoothing rate for Jacobi with optimal parameter $\bar{\omega}$ (generally different than $\omega$ ), applied to (5.1), exhibits a smoothing rate bounded by $\varepsilon^{1 / 4}$. This implies that four Jacobi sweeps on (5.1) reduce the oscillatory error better than one Jacobi sweep on (3.2). Since relaxation on (5.1) is significantly more expensive than on (3.2), the penalty of using (5.1) in lieu of (3.2) for a symmetric matrix $A^{h}$ is about an order of magnitude increase in computational cost. (The smaller the smoothing rate $\varepsilon$, the smaller is this penalty. Note that the actual penalty depends on the details of how the unigrid directions are implemented and how the operators $A^{h}$ and $\left(A^{h}\right)^{T}$ are accessed.) For matrices $A^{h}$ that deviate significantly from symmetry, the extra costs are correspondingly smaller to the point that unigrid on (5.1) will converge even though on (3.2) it may not. (Note the close relationship between (5.1) and the distributed relaxation schemes in [1]. In fact, the latter corresponds to a transformation of (3.2) via

$$
A^{h}\left(A^{h}\right)^{T} V^{h}=f^{h}, \quad U^{h}=\left(A^{h}\right)^{T} V^{h},
$$

which has some cost advantages with multigrid. With unigrid the costs are similar. The main difference with either method is that relaxation on (5.2) minimizes actual error, and on (5.1) it minimizes residual error.)

There are several advantages in using (5.1) as opposed to (3.2) besides generality. For example, not only is convergence of symmetric schemes better understood, but error control is better; that is, it is important that relaxation on (5.1) actually minimizes the residual in the $d^{h}$ direction. This is not true for (3.2) even when $A^{h}$ is symmetric and positive definite, although it should be approximately so. (In fact, when direct application of unigrid to (3.2) exhibits convergence but does not 
monotonically reduce the residual error on the coarse levels, this is a signal that the directions for relaxation are improperly defined. They should be chosen to approximate the smooth eigenvectors of $A^{h}$, that is, those that belong to the lower end of its spectrum. This would ensure that relaxation quickly eliminates the oscillatory eigenvector components of the error with little effect on the smooth ones. Since the spectrum of $A^{h}$ that corresponds to these oscillatory components is relatively narrow, then there is a close relationship between error in the energy norm, for which relaxation is a minimizer, and the residual error norm. The residual norm is not generally minimized by relaxation, but a proper choice of directions coupled with a good smoothing rate ensures that it will be monotonically reduced.)

Remark 3. A major advantage in using full unigrid (or full multigrid) is that the work required on a given level is fixed so that $\nu$ need only be sufficiently large to produce the required error reduction. This is easiest to see for symmetric positive definite $A^{h}$ by considering the level $h$ truncation error in the $\mathcal{H}$-energy norm given by

$$
\tau^{h}=\left\|I_{h} U^{h}-U\right\|_{A} .
$$

Here, $I^{h}: \mathcal{H} \rightarrow \mathcal{H}^{h}$ and $I_{h}: \mathcal{H}^{h} \rightarrow \mathcal{H}$ are the discretization operators that, together with $A$, satisfy a continuous analogue of the variational conditions (4.1)-(4.2). For any $u^{2 h} \in \mathcal{H}^{2 h}$, with error $e^{(2 h)}=I_{2 h} u^{2 h}-U$, then $u^{h}=I_{2 h}^{h} u^{2 h}$ has the same error $e^{(h)}=I_{h} u^{h}-U$, that is, $e^{(h)}=e^{(2 h)}$. Thus, if $u^{2 h}$ is an approximation to $U^{2 h}$ computed so that, say,

$$
\left\|u^{2 h}-U^{2 h}\right\|_{A^{2 h}} \leqslant \tau^{2 h}
$$

then

$$
\left\|e^{(2 h)}\right\|_{A} \leqslant 2 \tau^{2 h}
$$

from which it follows that

$$
\left\|u^{h}-U^{h}\right\|_{A^{h}}=\left\|I_{h} u^{h}-I_{h} U^{h}\right\|_{A} \leqslant\left\|I_{h} u^{h}-U\right\|_{A}=\left\|e^{(2 h)}\right\|_{A} \leqslant 2 \tau^{2 h} .
$$

If $\tau^{h}$ satisfies an expression like $\tau^{h} \cong c h^{2}$ and if $\nu$ is large enough to reduce the error $\left\|u^{h}-U^{h}\right\|$ by at least a factor of 8 , then full unigrid would ensure the desired result

$$
\left\|e^{(h)}\right\|_{A} \leqslant \tau^{h} .
$$

6. Multigrid Simulation. There are many potential uses for unigrid, perhaps as a method in its own right, but more importantly as a simulator to test the feasibility of using multigrid in a given application (especially when software already exists for solving the given problem). Attributes that favor unigrid include compact code, small storage requirements, ease of design and programming potential for vectorization and parallelism, and general guarantee of convergence. However, although it is more efficient than most methods, it is more costly than multigrid. Nevertheless, it is very useful as a multigrid simulator where it benefits from ease of implementation and automatic implementation of the variational conditions (4.1)-(4.2).

To apply unigrid to a given problem, the design question is mainly one of determining the directions in (3.5). Since a suitable choice for the interpolation operator is often dictated by the discretization process that produced the problem to be solved, then the only remaining decision is to choose the fine grid directions, that is, the relaxation process. Unigrid algorithm design is therefore a great simplification 
over that for multigrid since the latter also involves careful determination of the grid transfers, coarse grid operators, and scale factors.

Some applications may require more flexibility in choosing the grid transfer and coarse grid operators than unigrid provides. However, if the discretization process that relates $\mathcal{H}^{h}$ to $\mathcal{H}$ is done properly, then a corresponding process can be used to relate $\mathcal{H}^{2 h}$ to $\mathcal{H}^{h}$. That is, a proper discretization should dictate the interpolation process. For the symmetric case at least, in some sense the best choices for the coarse grid transfer and coarse grid operator are specified by the variational conditions (4.1)-(4.2). The same is true for the nonsymmetric case when unigrid is applied via the normal equations (5.1). Thus, in terms of the original problem solution, unigrid should provide a very effective design.

The inflexibility in unigrid design is precisely the same inflexibility inherent in Galerkin-type discretizations of (3.1). In fact, such discretization methods naturally satisfy (4.1)-(4.2). Unigrid automatically adopts this sort of relationship between grids regardless of how the finest grid was obtained.

There are many possibilities in the use of unigrid as a multigrid simulator. One approach is to design a portable black box that implements unigrid on a fixed region, such as a rectangle, and for a fixed relaxation method, such as Gauss-Seidel. This software developement utility could be easily plugged into an existing possibly very complex applications program, for example, that already uses some form of relaxation such as SOR. This would require no knowledge of the program data structure, problem origin, scaling factors or other information that multigrid implementation demands.

Another important use of unigrid as a multigrid simulator is as a research tool. The authors have developed many unigrid versions written in BASIC for the HP9845B, none of which required more than an hour of program development. Many research questions were dealt with quickly using these routines for experimentation. A few versions of these programs together with sample output are displayed in Tables 3 through 10.

The programs listed in Tables 3 and 5 apply to the problem

$$
\begin{aligned}
-\Delta u+g \cdot u=f & & \text { in } \Omega=(0, \alpha) \times(0, \beta), \\
u=b & & \text { on } \partial \Omega,
\end{aligned}
$$

where $\alpha, \beta$ are determined by the program input and the functions $f, g$ and $b$ are specified internally. This is similar to, but an extension of, the problem example reported in [1]. Here we use $g(x, y)=(x-y) e^{x+y-3}, f(x, y)=\sin (3(x+y))$, $b=\cos (3(x+y))$, and $(\alpha, \beta)=(3,2)$.

Table 3 lists the code for the full unigrid algorithm where cycles start from the coarsest level. This process progresses to the next finer level only after a full cycle is performed on the present finest level. (It is best to use 1 as the number of cycles since full unigrid will then produce an approximation to the level of truncation error provided $\nu$ is not too small. Usually $\nu=2$ or 3 will do. See Remark 3 of Section 5.) Note that the problem is a nine-point discretization of (6.1) and is defined in statements 480-530 and functions FNB, FNF and FNG. Modifications to these statements and functions can be easily made to apply this code to other problems. Table 4 depicts output from a sample run with full unigrid. 


\section{TABLE 3. Full unigrid code}

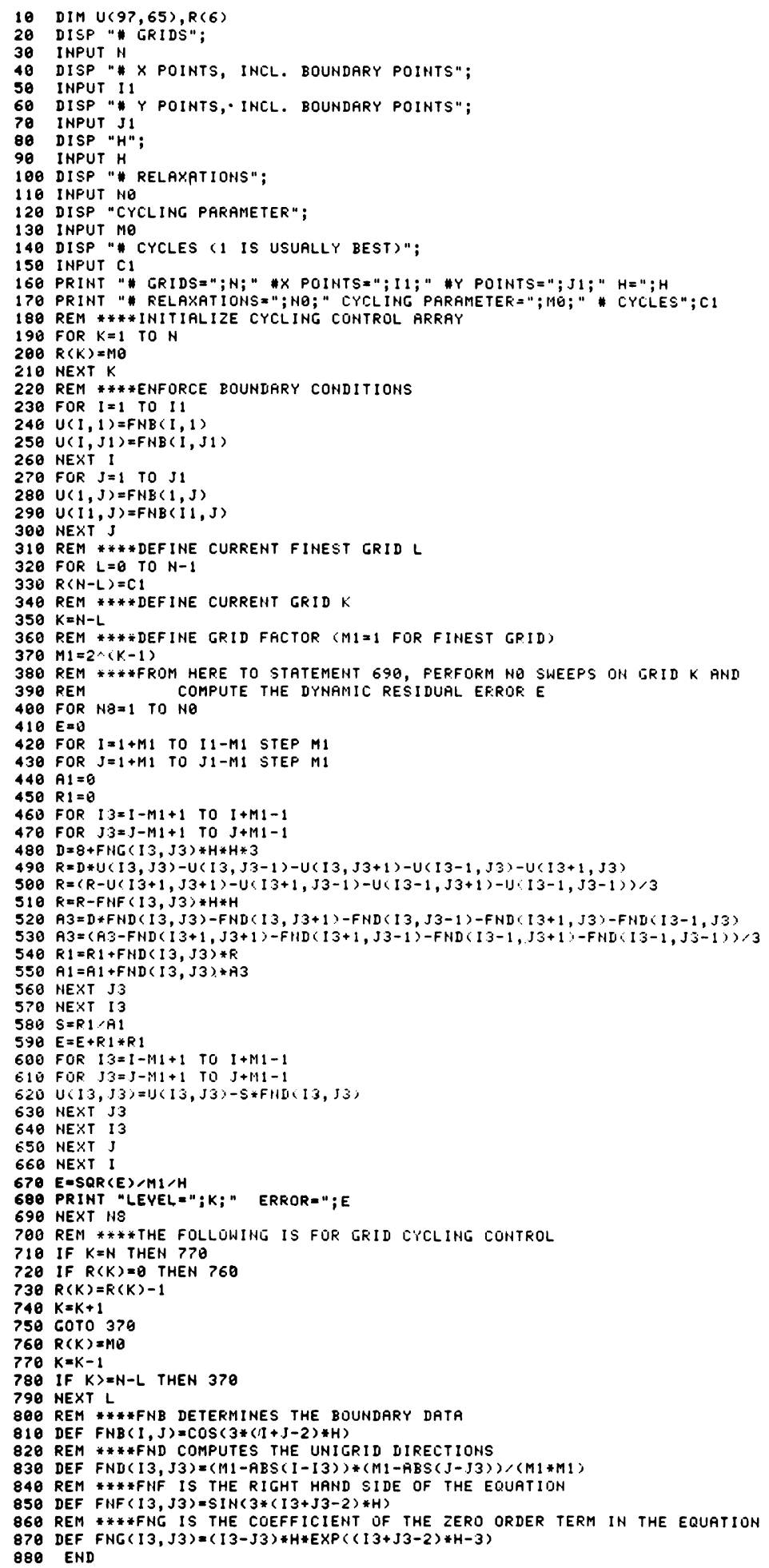


TABLE 4. Full unigrid output

\begin{tabular}{|c|c|c|c|}
\hline \multicolumn{4}{|c|}{$\begin{array}{l}\# G R I D S=4 \\
\# X=25 \quad \# y=17 \\
H=.125 \quad \# R E L X=2 \\
C Y C . P A R A M .=2 \quad \# C Y C=1\end{array}$} \\
\hline$L V L=4$ & $E R R=1.6 E 0$ & $L V L=3$ & $E R R=9,6 E-2$ \\
\hline$L V L=4$ & $E R R=1.0 E-1$ & $L V L=3$ & $E R R=2.2 E-2$ \\
\hline$L V L=3$ & $E R R=3.6 E 0$ & $L V L=4$ & $E R R=3.6 E-3$ \\
\hline$L V L=3$ & $E R R=5.2 E-1$ & $L V L=4$ & $E R R=2.9 E-4$ \\
\hline$L V L=4$ & $E R R=6.25-2$ & $L V L=3$ & $E R R=4.4 E-3$ \\
\hline$L V L=4$ & $E R R=5.0 E-3$ & $L V L=3$ & $E R R=9.8 E-4$ \\
\hline $\mathrm{LVL}=3$ & $E r r=7.15-2$ & $L V L=4$ & $E R R=1.5 E-4$ \\
\hline $\mathrm{LVL}=3$ & $E R R=1.3 E-2$ & $L V L=4$ & $E R R=6.5 E-9$ \\
\hline$L V L=2$ & $E R R=1.6 \mathrm{El}$ & $L V L=3$ & $E R R=1.4 \mathrm{E}-4$ \\
\hline$L V L=2$ & $E R R=2.7 E 0$ & $L V L=3$ & $E R R=2.4 E-5$ \\
\hline$L V L=3$ & $E R R=4.3 E-1$ & $L V L=2$ & $E R R=1.0 E-1$ \\
\hline $\mathrm{LVL}=3$ & $E R R=8.2 E-2$ & $L V L=2$ & $E R R=2.5 E-2$ \\
\hline $\mathrm{LVL}=4$ & $E R R=1.3 E-2$ & $L V L=3$ & $E R R=4.8 E-3$ \\
\hline$L V L=4$ & $E R R=9.5 E-4$ & $L V L=3$ & $E R R=1.1 E-3$ \\
\hline$L V L=3$ & $E R R=1.6 \mathrm{E}-2$ & $L V L=4$ & $E R R=1.0 E-4$ \\
\hline$L \mathrm{LL}=3$ & $E R R=3.4 E-3$ & $L V L=4$ & $E R R=9.4 E-6$ \\
\hline$L V L=4$ & $E R R=5.0 E-4$ & $L V L=3$ & $E R R=2.8 E-4$ \\
\hline $\mathrm{LVL}=4$ & $E R R=1.1 E-6$ & $L V L=3$ & $E R R=6.2 E-5$ \\
\hline $\mathrm{LVL}=3$ & $E R R=4.7 E-4$ & $L V L=4$ & $E R R=9.3 E-6$ \\
\hline$L V L=3$ & $E R R=7.6 E-5$ & $L V L=4$ & $E R R=2.4 E-8$ \\
\hline$L V L=2$ & $E R R=5.5 E-1$ & $L V L=3$ & $E R R=1.0 E-5$ \\
\hline LVL=2 & $E R R=1.2 E-1$ & $\mathrm{LVL}=3$ & $E R R=1.7 \mathrm{E}-6$ \\
\hline $\mathrm{LVL}=1$ & $E R R=4.9 E 7$ & $L V L=2$ & $E R R=5.1 E-3$ \\
\hline LVL=1 & $E R R=1.0 E I$ & $L V L=2$ & $E R R=1.2 E-3$ \\
\hline$L V L=2$ & $E R R=2.1 E O$ & $L V L=1$ & $E R R=1.8 E 0$ \\
\hline$L V L=2$ & $E R R=5.1 E-1$ & $L V L=1$ & $E R R=4.0 E-1$ \\
\hline
\end{tabular}

Table 5 contains the code for a very simple version of unigrid applied to the usual five-point discretization of (6.1). The listing shown produces the coarse-to-fine level cycling scheme depicted in column one of Table 4 . Note that the problem for this sample run has 5985 unknowns. To demonstrate the effect of fine-to-coarse level cycling, statements 240 and 510 were modified by replacing $N-K$ by $K-1$. Note the decrease in accuracy for this run. This is due to the smoothness of the initial error which for the first cycle or so favors coarse grid relaxations. Otherwise, the two cycling schemes behave almost identically as is evidenced by the convergence rates of the last few cycles.

Statements 170-210 in the sample code of Table 5 define the boundary and the initial guess for unigrid. The corresponding statements 220-300 for the full unigrid code of Table 3 define the boundary but yield zero as an initial guess (for operating systems like the HP9845B, at least). This was done to mimic the full multigrid algorithm which is usually implemented when a good guess for the grid solution is unavailable. Had this code incorporated the same statements as in Table 3, it would in effect be applying full unigrid to the fine grid residual equation

$$
A^{h} U^{h}=\bar{f}^{h} \text {, }
$$

where $\bar{f}^{h}=f^{h}-A^{h} b^{h}$ and $b^{h}$ is the initial guess provided by these statements. (In fact, use of full unigrid is often advised even when $b^{h}$ is a good approximation to $U^{h}$, such as for implicit discretization of time-dependent problems. This is especially true for extensions to nonlinear problems.) 
TABLE 5. Simple cycle unigrid code

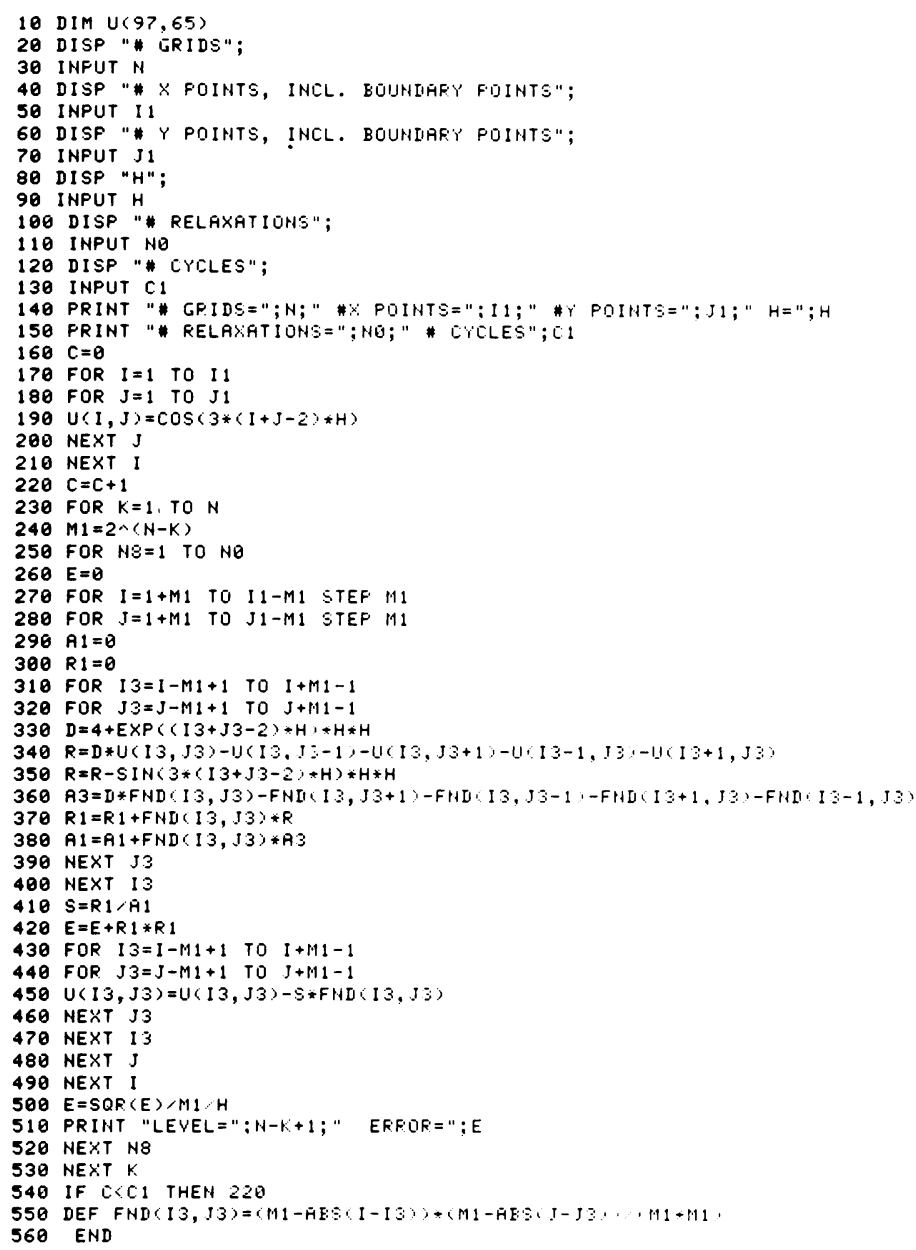

To estimate the computational complexity of a multigrid algorithm corresponding to these unigrid codes, work units (defined as in [1] as the cost of one fine grid relaxation sweep) can be accumulated for output. For Table 5, this could be done by adding statements

$$
\begin{array}{ll}
165 & W=0 \\
255 & W=W+(1.0 / M 1) \\
545 & \text { PRINT “TOTAL WORK = "; } W .
\end{array}
$$

For the run in column one of Table 6 this would yield

$$
\text { TOTAL WORK }=8 \text {. }
$$

These codes determine and output the dynamic residual error norm using the discretized $\mathcal{L}_{2}(\Omega)$ norm on the grid corresponding to the level in use. However, unigrid and variationally formulated multigrid are based on minimizing the energy norm of the actual error, which is equivalent to minimizing the functional

$$
F^{h}\left(u^{h}\right)=\left\langle A^{h} u^{h}, u^{h}\right\rangle-2\left\langle f^{h}, u^{h}\right\rangle .
$$


TABLE 6

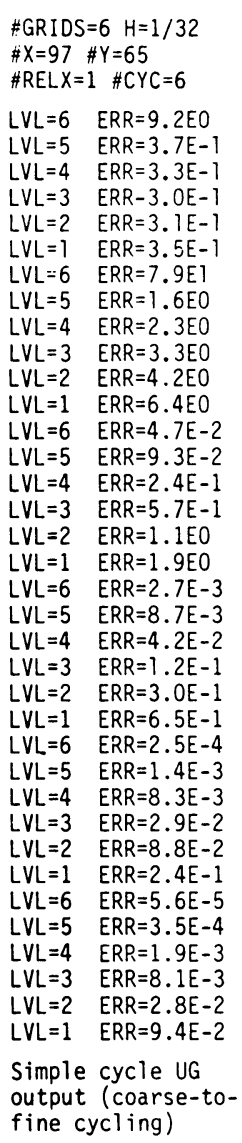

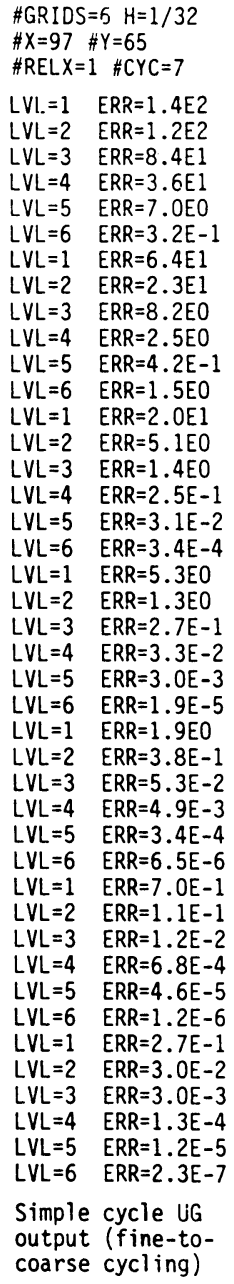

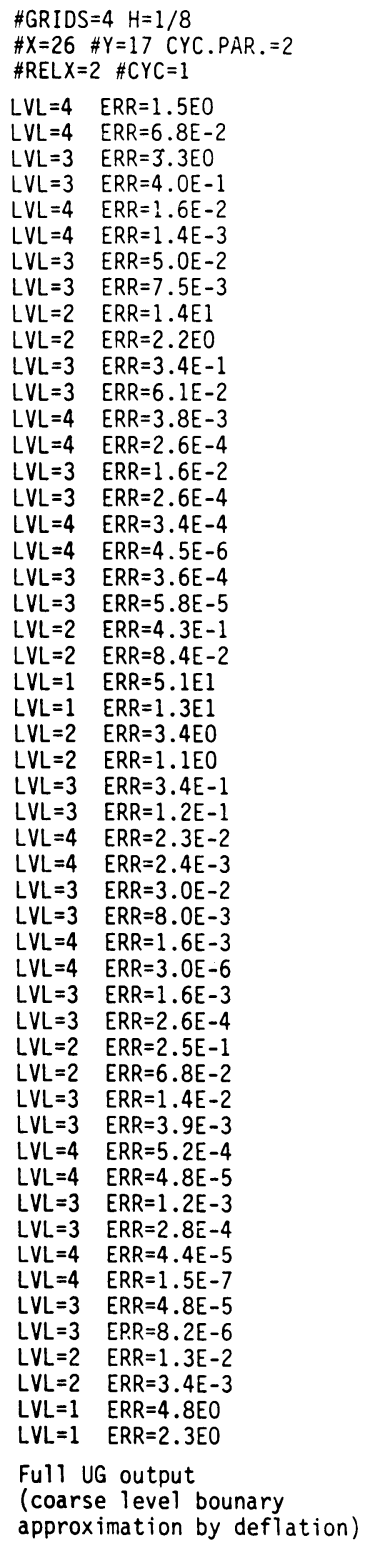

In fact, the square of the energy norm error is just $F^{h}\left(u^{h}\right)-F^{h}\left(U^{h}\right) .\left(F^{h}\left(U^{h}\right)\right.$ may be estimated by simple extrapolation of the $F^{h}\left(u^{h}\right)$.) It may therefore be more practical to compute this functional at the end of each relaxation sweep, expecially since it will more clearly represent the convergence behavior of the algorithm. This can be done in just a few statements and is especially useful for program debugging. (The reader is warned to be sure that the boundary conditions are incorporated in $f^{h}$.) 


\section{TABLE 7. Full unigrid code}

(modified for coarse level boundary approximation by direction truncation)

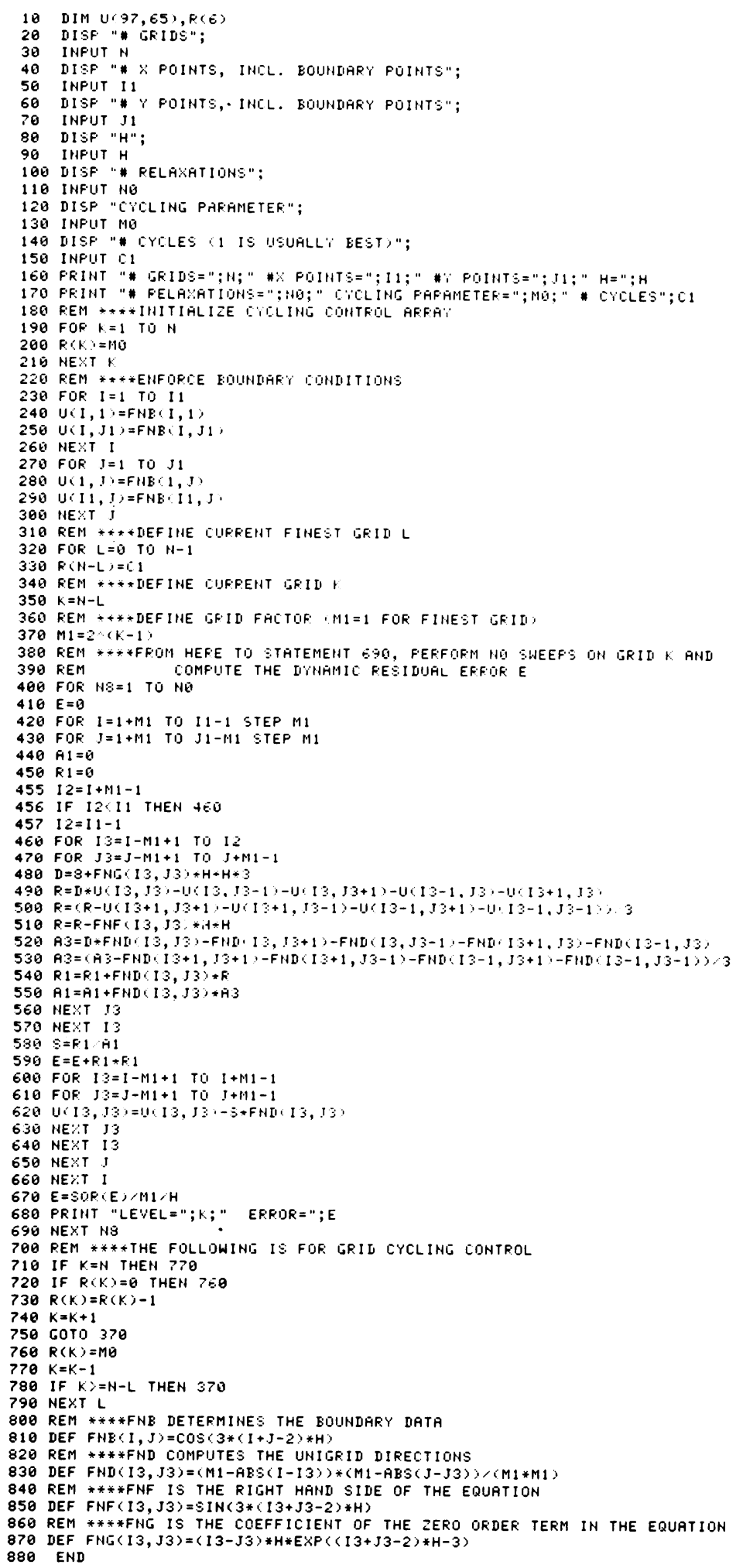


TABLE 8. Full unigrid output

(coarse level boundary approximation by direction truncation)

\begin{tabular}{|c|c|}
\hline$L V_{L}=4$ & $\begin{array}{l}E R R=3.5 E 0 \\
E R R=8.8 E-2\end{array}$ \\
\hline$L V L=3$ & $\begin{array}{l}E R R=8.8 E-2 \\
E R R=5.5 E 0\end{array}$ \\
\hline$L V L=3$ & $\begin{array}{l}E R R=5.5 E 0 \\
E R R=6.4 E-1\end{array}$ \\
\hline$L V L=4$ & $\begin{array}{l}E R R=6.4 E-1 \\
E R R=4.6 E-2\end{array}$ \\
\hline$L V L=4$ & $\begin{array}{l}E R R=4.6 E-2 \\
E R R=3.1 E-3\end{array}$ \\
\hline LVL-3 & $\begin{array}{l}E R R=3.1 E-3 \\
E R R=7.2 E-2\end{array}$ \\
\hline$L V L=3$ & $\begin{array}{l}E R R=7.2 E-2 \\
E R R=7.6 E-3\end{array}$ \\
\hline$L V L=2$ & $\begin{array}{l}E R R=7.6 \mathrm{E}-3 \\
E R R=1.8 \mathrm{E} 1\end{array}$ \\
\hline LVL-2 & $\begin{array}{l}E R R=1.8 E 1 \\
E R R=2.5 E 0\end{array}$ \\
\hline$L V L=3$ & $\begin{array}{l}E R R=2.5 E 0 \\
E R R=3.7 E-1\end{array}$ \\
\hline$L V L=3$ & $\begin{array}{l}E R R=3.7 E-1 \\
E R R=6.4 E-2\end{array}$ \\
\hline$L V L=4$ & $\begin{array}{l}E R R=6.4 E-2 \\
E R R=2.4 E-3\end{array}$ \\
\hline $\mathrm{LVL}=4$ & $\begin{array}{l}E R R=2.4 E-3 \\
E R R=1.4 E-4\end{array}$ \\
\hline$L V L=3$ & \\
\hline$L V L=3$ & $\begin{array}{l}E R R=1.6 E-2 \\
E R R=2.7 E-3\end{array}$ \\
\hline$L V L=4$ & $\begin{array}{l}E R R=2.7 E-3 \\
E R R=3.8 E-4\end{array}$ \\
\hline$L V L=4$ & $\begin{array}{l}E R R=3.8 E-4 \\
E R R=3.9 E-6\end{array}$ \\
\hline$L V L=3$ & $\begin{array}{l}E R R=3.9 E-6 \\
E R R=4.4 E-4\end{array}$ \\
\hline$L V L=3$ & \\
\hline$L V L=2$ & $\begin{array}{l}E R R=7.4 E-5 \\
E R R=4.5 E-1\end{array}$ \\
\hline$L V L=2$ & $\begin{array}{l}E R R=4.5 E-1 \\
E R R=8.8 E-2\end{array}$ \\
\hline$L V L=1$ & $\begin{array}{l}E R R=8.8 E-2 \\
E R R=4.6 E 1\end{array}$ \\
\hline$L V L=1$ & \\
\hline$L V L=2$ & $\begin{array}{l}E R R=7.7 E 0 \\
E R R=1.4 E 0\end{array}$ \\
\hline$L V L=2$ & $\begin{array}{l}E R R=1.4 E 0 \\
E R R=3.2 E-1\end{array}$ \\
\hline$L V L=3$ & $\begin{array}{l}E R R=3.2 E-1 \\
E R R=6.2 E-2\end{array}$ \\
\hline$L V L=3$ & \\
\hline$L V L=4$ & $\begin{array}{l}E R R=1.3 E-2 \\
E R R=7.9 E-5\end{array}$ \\
\hline$L V L=4$ & $\begin{array}{l}E R R=7.9 E-5 \\
E R R=1.3 E-6\end{array}$ \\
\hline$L V L=3$ & \\
\hline $\mathrm{LVL}=3$ & $\begin{array}{l}E R R=3.1 E-3 \\
E R R=5.6 E-4\end{array}$ \\
\hline$L V L=4$ & $\begin{array}{l}E R R=5.6 E-4 \\
E R R=7.1 E-5\end{array}$ \\
\hline$L V L=4$ & $\begin{array}{l}E R R=7.1 E-5 \\
E R R=5.1 E-7\end{array}$ \\
\hline$L V L=3$ & $\begin{array}{l}E R R=5.1 E-7 \\
E R R=8.4 E-5\end{array}$ \\
\hline$L V L=3$ & $\begin{array}{l}E R R=8.4 E-5 \\
E R R=1.5 E-5\end{array}$ \\
\hline$L V L=2$ & $\begin{array}{l}E R R=1.5 E-5 \\
E R R=6.4 E-2\end{array}$ \\
\hline$L V L=2$ & $\begin{array}{l}E R R=6.4 E-2 \\
E R R=1.5 E-2\end{array}$ \\
\hline$L V L=3$ & $\begin{array}{l}E R R=1.5 E-2 \\
E R R=3.1 E-3\end{array}$ \\
\hline$L V L=3$ & $\begin{array}{l}E R R=3.1 E-3 \\
E R R=7.6 E-4\end{array}$ \\
\hline$L V L=4$ & $\begin{array}{l}E R R=7.6 E-4 \\
E R R=16 E-5\end{array}$ \\
\hline $\begin{array}{l}\mathrm{LVL}=4 \\
\text { L }\end{array}$ & $E R R=1.6 E-5$ \\
\hline$L V L=3$ & $E R R=1.3 E-6$ \\
\hline$L V L=3$ & $E R R=1.8 E-4$ \\
\hline$L V L=4$ & $E R R=2.7 E-5$ \\
\hline$L V L=4$ & $E R R=3.7 E-6$ \\
\hline$L V L=3$ & $\begin{array}{l}E R R=4.7 E-8 \\
E R R=4.4 E-6\end{array}$ \\
\hline$L V L=3$ & $\begin{array}{l}E R R=4.4 E-6 \\
E R R=7.1 E-7\end{array}$ \\
\hline $\mathrm{LVL}=2$ & $E R R=3.1 E-3$ \\
\hline$L V L=2$ & $E R R=6.7 E-4$ \\
\hline$L V L=1$ & $E R R=1.5 E 0$ \\
\hline$L V L=1$ & $E R R=3.0 E-1$ \\
\hline
\end{tabular}

The codes listed in Tables 3 and 5 are intended neither for efficiency nor structure. In fact, the runs depicted in Table 6 required several hours of HP9845B execution time. The main goal was ease of programming and of program modification. Dr. Bill Holland of the National Center for Atmospheric Research has a very well-developed version of unigrid that uses stencils to define the directions and is extensively modularized and structured. It is implemented on his Apple home computer and is being used to test the applicability of unigrid and multigrid to the time-dependent irregular domain diffusion problems that arise in atmospheric studies. 
TABLE 9. Nonlinear simple cycle unigrid code

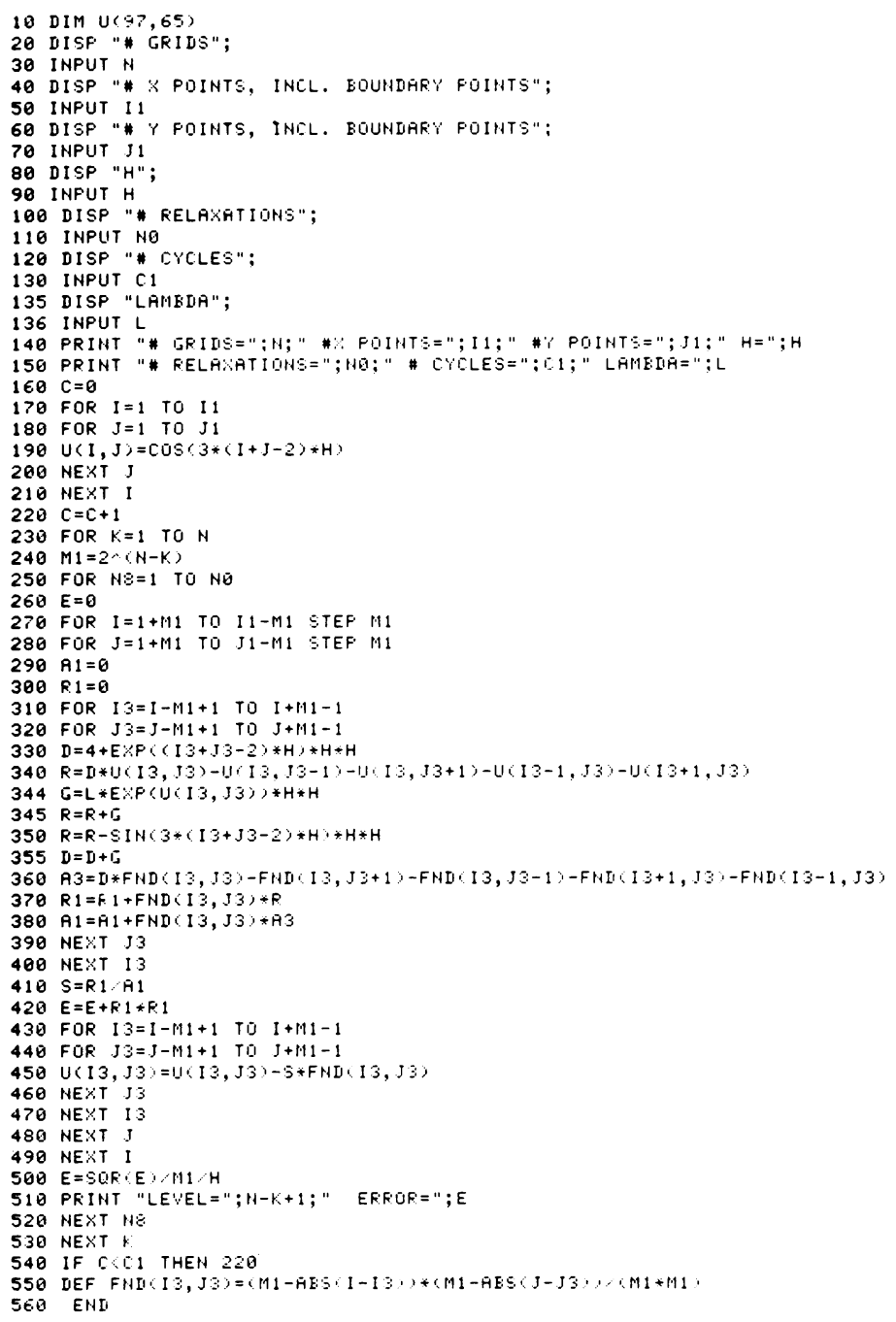

7. Sample Uses. This section illustrates, by way of two examples, the ease of use of unigrid as a research tool to investigate design questions for multigrid application to a given problem.

Discretizations of nonrectangular regions often have the property that the boundary lies along lines of the grid on which the problem is to be resolved. This is a computational advantage, especially when vector computers are to be used. Unfortunately, the coarser grids needed for multigrid application do not generally share this grid alignment feature unless the coarser boundaries are subject to approximation. 
TABLE 10

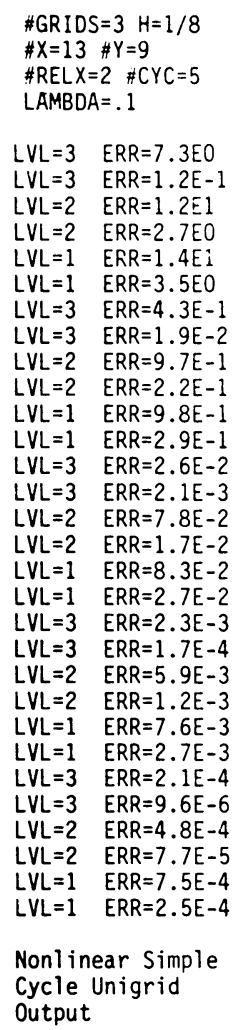

\#GRIDS=3 $\quad H=1 / 8$

$X=13 \# Y=9$

$\angle A M B D A=.1$

$L V L=3 \quad E R R=7.3 E 0$

$L V L=3 \quad E R R=1.2 E-1$

$L V L=2 \quad E R R=2.7 E 0$

$L V L=1 \quad E R R=1.4 E 1$

$L V L=1 \quad E R R=3.5 E 0$

$L V L=3 \quad E R R=4 \cdot 3 E-1$

$L V L=2 \quad E R R=9.7 E-1$

$L V L=2 \quad E R R=2.2 E-1$

$L V L=1 \quad E R R=9.8 \mathrm{E}-1$

$L V L=3 \quad E R R=2.6 E-2$

$L V L=3 \quad E R R=2.1 E-3$

$L V L=2 \quad E R R=7.8 E-2$

$L V L=2 \quad E R R=1.7 E-2$

$L V L=1 \quad E R R=8.3 E-2$

$L V L=3 \quad E R R=2.3 E-3$

$L V L=3 \quad E R R=1.7 E-4$

$L V L=2 \quad E R R=5.9 E-3$

$L V L=2 \quad E R R=1.2 E-3$

$L V L=1 \quad E R R=7.6 E-3$

$L V L=3 \quad E R R=2.1 E-4$

$L V L=3 \quad E R R=9.6 E-6$

$L V L=2 \quad E R R=4.8 E-4$

$L V L=2 \quad E R R=7.7 E-5$

$L V L=1 \quad E R R=7.5 E-4$

Nonl inear Simple

Output

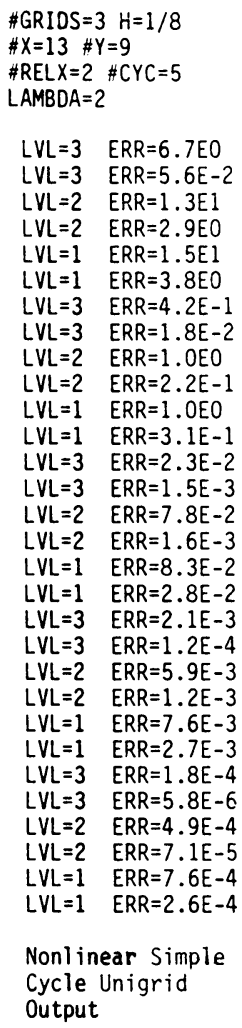

One possible grid alignment approximation scheme is to collapse the boundary onto grid lines and use as boundary date the (weighted) values of the finer grid solution approximation at the points on which the boundary is collapsed. To test the effect of this approach with unigrid, it is enough to specify a number of grid points in one or both directions that is not of the form $2^{m}+1$, where $m$ is the number of grids. For the sample run in column 3 of Table 6 , the number of $x$ points is 26 which means that the exact right boundary on level 2 is a half grid size from coarse grid points. Statement 420 in Table 3 therefore has the effect of ignoring those directions that extend beyond this boundary. The resulting efficiency as depicted in column 3 of Table 6 is significantly degraded over the similar run depicted in Table 4.

Another possibility is to expand the boundary to align it properly with the coarse grid. This is done in multigrid by defining the interpolation operator that is natural for relating these grids. For unigrid this is done simply by clipping the directions where they overlap the fine grid region. Table 7 illustrates a unigrid code modified for this purpose (with new statements $455-457$ and changes to 420 and 460). A sample run is shown in Table 8 . Note that this version of multigrid fully restores the efficiency observed in Table 4. 
The second sample use of unigrid is illustrated by the code in Table 9. This program is a modification of that in Table 5 that applies to the nonlinear problem

$$
\begin{aligned}
-\Delta u+\lambda e^{u} & =f & & \text { in } \Omega=(0, \alpha) \times(0, \beta), \\
u & =b & & \text { on } \partial \Omega .
\end{aligned}
$$

Sample runs with $\lambda=.1$ and $\lambda=2$ are in columns 1 and 2, respectively, of Table 10 .

The total human effort for achieving the results described in this section was less than one man-hour.

Acknowledgement. This work was supported by the National Science Foundation under grant number MCS78-03847 and the Air Force Office of Scientific Research under grant number F33615-79-C-3223.

Department of Mathematics
Colorado State University
Fort Collins, Colorado 80523

1. A. Brandt, "Multilevel adaptive solutions to boundary value problems”, Math. Comp., v. 31, 1977. pp. 333-390.

2. S. F. McCormick \& J. W. Ruge, “Multigrid for variational problems," SIAM J. Numer. Anal., v. 19. 1982, pp. 924-929. 